\title{
The use of nasal trumpet as a non-invasive treatment method in congenital nasal stenosis
}

\author{
Özgür Kemal ${ }^{1}$, Sinan Atmaca ${ }^{1}$, Ayşe Bel-Çeçen ${ }^{1}$, Berkan Düzgün${ }^{1}$, Hasibe Canan Aygün${ }^{2}$ \\ Departments of ${ }^{1}$ Otolaryngology, ${ }^{2}$ Pediatrics, Ondokuz Mayıs University Faculty of Medicine, Samsun, Turkey. \\ E-mail: drozgurkemal@gmail.com \\ Received: 26th September 2016, Revised 6th October 2016, Accepted: 6th January 2017
}

\begin{abstract}
SUMMARY: Kemal Ö, Atmaca S, Bel-Çeçen A, Düzgün B, Aygün HC. The use of nasal trumpet as a non-invasive treatment method in congenital nasal stenosis. Turk J Pediatr 2017; 59: 210-213.

Newborns and infants are dependent on nasal respiration and therefore, nasal obstructions may lead to life-threatening outcomes in this age group. Although the most common cause of nasal obstruction in newborns are choanal atresia, soft tissue edema, congenital nasal bone anomalies such as narrowness of the apertura piriformis and midnasal stenosis should be kept in mind.

A 5-day-old infant was referred to our clinic with the complaints of respiratory problems. Physical examination revealed nasal bone hypoplasia and saddle nose deformity. In the endoscopic examination, the septum was seen to be deviated in an ' $S$ ' shape and the nasal passages were almost completely closed. The paranasal CT examination reported significant narrowing of the airway in the central part of the nasal cavity. After application of nasal trumpet to both nasal passages, clinical recovery was determined in the patient and no pathology was determined during the follow-up period.
\end{abstract}

Key words: nasal stenosis, nasal trumpet, newborn.

As newborns are dependent on nasal respiration, any pathology preventing the normal airflow must be diagnosed quickly and the necessary precautions should be taken.

Although structural nasal anomalies as less common, choanal atresia and pyriform aperture stenosis are seen relatively more frequently, more uncommon anomalies such as midnasal stenosis must not be ignored. Before surgical treatment for midnasal stenosis, there are conservative treatments such as nasal drops and McGovern pacifier in addition to the treatment option of nasal airway which is non-invasive, low-cost, and can be applied easily and quickly ${ }^{1}$.

There have been extremely few cases of midnasal stenosis and treatment reported in literature. Raghavan et $\mathrm{al}^{2}$, reported treatment of dilatation and nasal stent application under general anesthesia in a 6-week old female Asian infant with bilateral midnasal stenosis. In the current case of a patient with midnasal stenosis, nasal trumpet application was considered to be an alternative treatment option that was non-invasive, low-cost and could be applied easily. The aim of this paper was to share this experience. Family compliance can be considered to be extremely good because of the ease of application of nasal trumpet use.

\section{Case Report}

A 5-day old male infant, born at 37 weeks, was referred to our clinic with complaints of respiratory problems which had been ongoing since birth and became more evident during feeding. In the antenatal ultrasonographic examination applied in the 5 th month of pregnancy, a nasal anomaly had been determined. Oxygen support by a hood was provided during the examination of the infant which revealed hypoplasia in the nasal bones and the collapse of the nasal root (Fig. 1).

Intercostal and suprasternal contractions were observed. The finest feeding tube was able to pass into both nasal passages. On paranasal tomography images, evident narrowing was observed in the airway at the level of the inferior turbinate in the central section of the nasal cavity (Fig. 2). 
As bruising and carbon dioxide retention developed in the patient during follow-up, surgery was planned. Endoscopic examination was done under general anesthesia. The septum was seen to be deviated to the right and there was narrowness in the central section of both nasal passages. Passages were seemed to be almost completely closed. A no. 3 intubation tube was placed in both nasal passages and the anterior edges were fixed to the septum with 4.0 prolene sutures. During follow-up, after the first month, the patient tore the septum

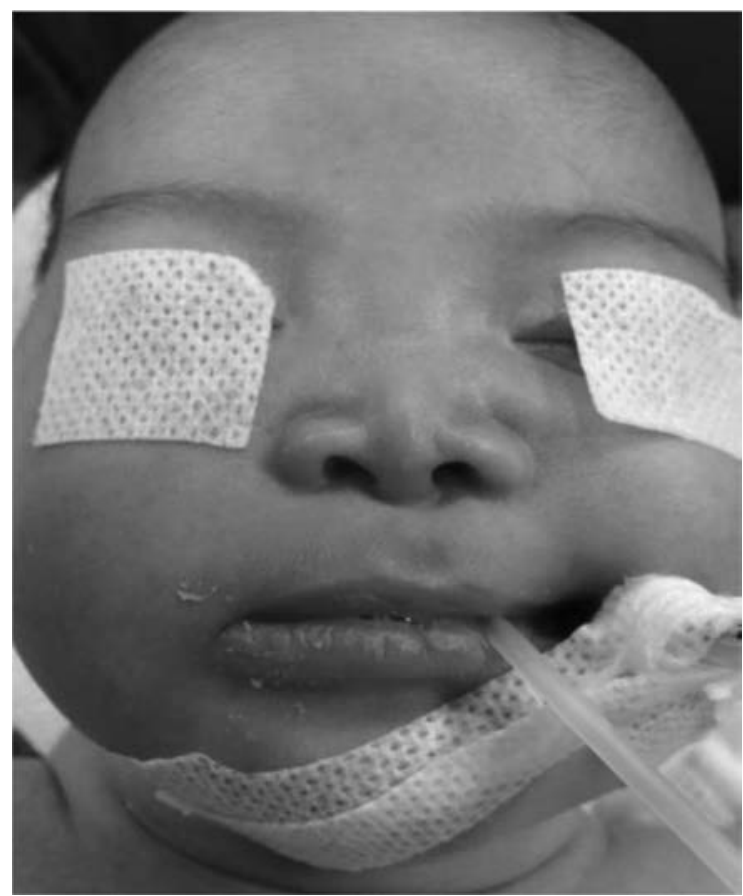

Fig. 1. The examination of the infant which revealed hypoplasia in the nasal bones and that the nasal root had collapsed.

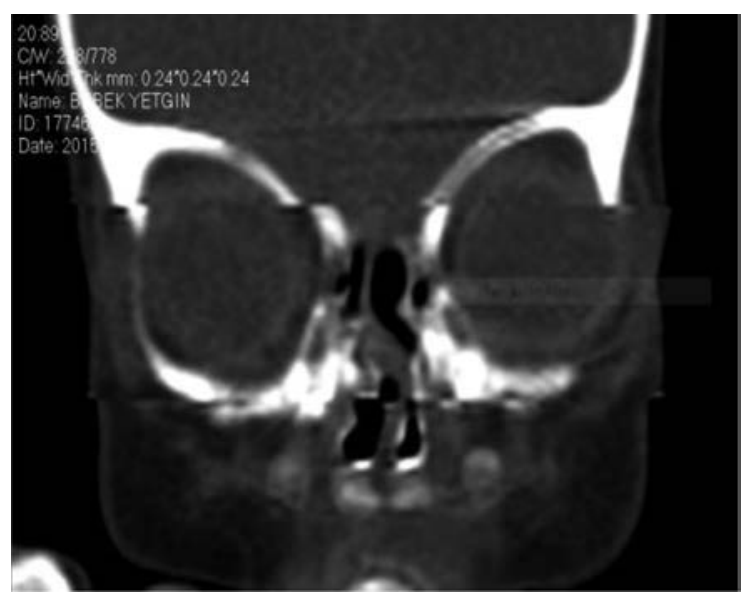

Fig. 2. CT-scan of midnasal stenosis. by pulling on the tubes, respiratory problems developed rapidly and so the intubated patient was again referred to our clinic. Bedside application of a no. 12 nasal airway (nasal trumpet) (Fig. 3) to both nasal passages was made and after a short period the patient was extubated. Nasal trumpet can be placed in the nose which can easily done by the parents and this is the advantage of the device. Although the risk of nasal trumpet removal by the infant exists, parents can insert them readily. The possibility of bilateral removal of the trumpets by the infant stipulates close parent or caregiver supervision. It is not fixed by a suture or something similar. When the patient was seen to have recovered well, he was discharged. Airways were used continuously for 1.5 months. At the follow-up examination 1.5 months later, the patient was seen to have no further need for the nasal trumpet.

We informed the patient about the publication and written informed consent was obtained

\section{Discussion}

The epiglottis in newborns extends as far as the soft palate, separating the digestive and respiratory tracts and thus feeding and respiratory functions can occur at the same time. Approximately $90 \%$ of newborns cannot achieve successful oral/nasal respiration coordination. This explains the dependence of the newborn on nasal respiration for the first 2 months of life $^{3}$. As newborns and infants are dependent on nasal respiration, findings of a nasal obstruction in this age group in

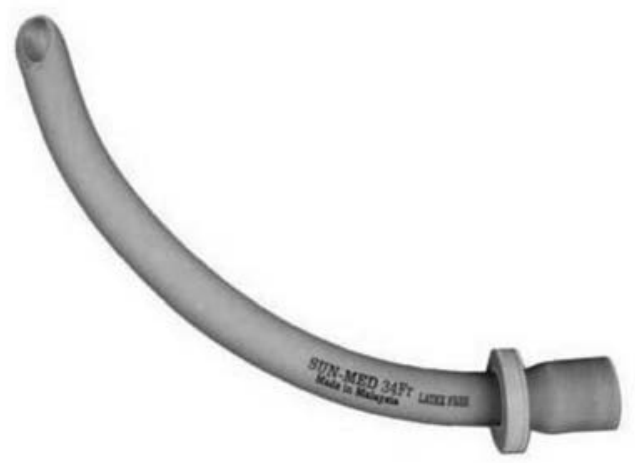

Fig. 3. Nasal airway (nasal trumpet). 
particular can be life-threatening and require emergency intervention. Nasal obstruction in newborns is identified by apnea seizures during feeding, chronic unilateral or bilateral rhinorrhea and findings of severe respiratory distress despite a normal tracheobronchial tree ${ }^{4}$. In addition, respiratory problems caused by a nasal obstruction in newborns show a tendency to be relieved by crying 5 . A case of rhinitis observed in this age group, non-specific nasal mucosa is the most frequently seen cause of nasal obstruction ${ }^{6}$. This condition generally responds to treatment with nasal saline drops administered in saline and will improve over time ${ }^{6}$.

The most serious results of nasal obstruction emerge associated with bone and fibrous tissue anomalies in the nasal cavity ${ }^{6}$. These are generally choanal atresia, piriform aperture stenosis and nasal cavity stenosis ${ }^{6}$. Tumoral formations such as glioma, encephalocele, teratoma and dermoid can be included in the less common reasons. Tomography is useful in the determination of the location and degree of nasal obstruction.

In the exclusion of choanal atresia and piriform aperture stenosis, care must be taken that midnasal stenosis is not overlooked in patients with nasal obstruction. Midnasal stenosis is narrowness of the fibrous or bone tissue in the nasal cavity between the piriform aperture and the choana 7 . This narrowness, which is generally unilateral, originates from disproportionate development of the lateral wall of the nasal cavity or advanced septum deviation ${ }^{7}$. In patients with nasal obstruction-related respiratory distress, the McGovern pacifier and oral airway can be used as conservative treatment ${ }^{1}$. Indications for surgical treatment in cases of nasal obstruction are nasal airway obstruction leading to sleep apnea, a history of repeated intubation or intolerance of intubation, feeding difficulties with concomitant cyanosis or the failure of the conservative treatments applied ${ }^{8}$. If a $5 \mathrm{Fr}$ catheter cannot be passed down the nasal passage, this is also an indication for surgical treatment ${ }^{9}$. In cases that do not respond to conservative treatment in a period of 10-15 days, surgery is recommended ${ }^{9}$.

Raghavan et al. ${ }^{2}$ reported the case of a 6 -week old Asian female infant with bilateral nasal stenosis. In this case, bone narrowness of 3 $\mathrm{cm}$ in length started from $0.5 \mathrm{~cm}$ posterior to the piriform aperture. After dilatation, stent placement was attempted. After 8 weeks the stent was removed and no recurrence of the complaints was observed during a 6-month follow-up period 2 . Özdemir et al. ${ }^{10}$ used balloon dilatation for a newborn with nasal stenosis and they offered their method as an alternative of surgery. They remarked that nasal balloon dilatation is an easy, non-invasive and repeatable way for nasal stenosis. In the current case, the application of nasal trumpet for midnasal stenosis was considered an extremely suitable treatment option as it does not require general anesthesia, can be applied easily at the bedside and has high family compliance.

In conclusion, in patients with findings of nasal obstruction who do not respond to medical treatment and for whom piriform aperture stenosis and choanal atresia have been excluded, midnasal stenosis should be kept in mind. The application of nasal trumpet to patients with midnasal stenosis can be accepted as a noninvasive, low-cost treatment method, which can be applied quickly and easily.

\section{REFERENCES}

1. Knegt-Junk K, Bos CE, Berkovits RN. Congenital nasal stenosis in neonates. J Laryngol Otol 1988; 102: 500502.

2. Raghavan U, Fuad F, Gibbin KP. Congenital midnasal stenosis in an infant. Int J Pediatr Otorhinolaryngol 2004; 68: 823-825.

3. Şenocak D. Pediyatrik üst solunum yolu obstrüksiyonu. Klinik Gelişim 2005; 18: 87-90.

4. Chinwuba C, Wallman J, Strand R. Nasal airway obstruction: CT assessment. Radiology 1986; 159: 503-506.

5. Ey EH, Han K, Towbin RB, Jaun WK. Bony inlet stenosis as a cause of nasal airway obstruction. Radiology 1988; 168: 477-479.

6. Jones JE, Jacqueline EJ, Oung E, Heier L. Congenital bony nasal cavity deformities. Am J Rhinol 1998; 12: 81-86.

7. Hall RK, Bankier A, Alfred MJ, Kan K, Lucas JO, Perks AG. Solitary median maxillary central incisor, short stature, choanal atresia/midnasal stenosis (SMMCI) syndrome. Oral Surg Oral Med Oral Pathol Oral Radiol Endod 1997; 84: 651-662.

8. Lee KS, Yang CC, Huang JK, Chen YC, Chang KC. Congenital pyrifirm aperture stenosis: surgery and evaluation with three-dimensional computed tomography. Laryngoscope 2002; 112: 918-921. 
9. Abbeele TVD, Triglia JM, Francois M, Narcy P. Congenital nasal pyriform aperture stenosis: diagnosis and management of 20 cases. Ann Otol Rhinol Laryngol 2001; 110: 70-75.
10. Özdemir R, Erdeve O, Süslü N, et al. Treatment of congenital nasal cavity stenosis by balloon dilatation in a newborn: A case report. Int J Pediatr Otorhinolaryngol 2011; 75: 960-962. 